\title{
Patient satisfaction and treatment outcomes as quality indicators for mental health services
}

\author{
Patricia Byrd Howard ${ }^{1}$, Peggy El-Mallakh², Mary Kay Rayens ${ }^{3}$ and \\ James J. Clark ${ }^{4}$
}

${ }^{1}$ Associate Professor, College of Nursing, University of Kentucky, Chandler Medical Center, 760 Rose Street, Lexington, Kentucky 40536-0232, USA, email pbhowa00@uky.edu, and Principal Investigator of the Kentucky Behavioral Health Monitoring Project

${ }^{2}$ Research Assistant, College of Nursing, University of Kentucky

${ }^{3}$ Associate Professor, College of Nursing, University of Kentucky

${ }^{4}$ Associate Professor, College of Social Work, University of Kentucky

n the United States, the patient has emerged as the central focus in evaluations of mental health services (Buckley, 1993). Whereas evaluation research in the $1980 \mathrm{~s}$ emphasised the structure and process of mental health care, current evaluation research incorporates clientbased measurements of treatment outcomes, such as symptom reduction, functional status and quality of life (Chisholm et al, 1997; Campbell, 1998). In addition, patient satisfaction with mental health services is increasingly used as an outcome dimension and an indicator of service quality (Center for Mental H ealth Services, 1996; Teague et al, 1997; Howard et al, 2003).

The purpose of this study was to investigate patient perspectives on service quality, satisfaction with services and mental health treatment outcomes in two publicsector psychiatric ho spitals in a south-eastern state.

\section{Method}

A simple survey design with a non-random sampling technique was used at two hospitals (designated 1 and 2 below). The sample consisted of eligible patients admitted during the study period who agreed to participate in the study. Inclusion criteria were:

o 18 years of age or older

O currently hospitalised

O identification by the treatment team as being symptomatically stable and near discharge from the hospital. Exclusion criteria were:

0 inability to give informed consent

o physical condition that precluded participation

o forensic classification.

\section{Data collection}

Research associates collected data at both hospitals. The research associates had received mental health services but were stable and living in the community at the time of the study. Inclusion of those who had received services in the role of data collectors enhanced the validity of the study findings (H oward \& El-M allakh, 2001). At hospital 1 the research associates were supervised by graduate students from the sponsoring university's College of
$\mathrm{N}$ ursing, and at hospital 2 by the risk management staff and patient advocate.

Sample characteristics

A total of 215 patients participated in the study: 107 participants from hospital 1 and 108 from hospital 2. Of these, 204 were acceptable for analysis: 103 from hospital 1 and 101 from hospital 2. The majority of the 204 respondents in the sample were male $(60.8 \%) ; 84.8 \%$ were European-American and the remainder were African-American. The mean age of the participants was 37.4 years $(s . d .=11.1)$. The most prevalent psychiatric diagnoses among respondents were major depression $(42.7 \%)$, schizophrenia $(25.0 \%)$ and substance misuse $(11.8 \%)$. Less frequent psychiatric diagnoses included delusion/other psychoses (4.9\%), Alzheimer's/organic brain disorders $(3.9 \%)$, impulse control disorders $(3.4 \%)$ and anxiety disorders (2.5\%). The majority of participants (63.7\%) were admitted to the hospital on an involuntary basis.

Instruments

Study instruments included the 19-item Kentucky Consumer Satisfaction Instrument (KY-CSI), the 21-item Mental $\mathrm{H}$ ealth Statistics Improvement Program (MHSIP) Consumer Survey, the 8-item Consumer Satisfaction $\mathrm{Q}$ uestionnaire (CSQ -8) and the single-item Q uality of Mental $\mathrm{H}$ ealth Care (Q MHC) instrument. The KY-CSI is a multi-dimensional scale that measures respondents' satisfaction with the physical environment of care, affliation with staff, family and other patients, and goal attainment/ self-actualisation (H oward et al, 2001). $O$ ne additional item questions whether the respondent would return to the facility if mental health services were needed in the future. The MHSIP Consumer Survey is a multidimensional scale that measures perceptions of access to care, appropriateness of mental health services, outcomes of treatment and general satisfaction with services (Ganju, 1999; Center for Mental Health Services, 1996). The CSQ - 8 measures global satisfaction with health services (Attkinson \& Greenfield, 1996). The single-item Q MHC measures respondents' overall perception of the quality of mental health care; response choices range from 0 ('worst possible care') to 10 ('best possible care').
Patient satisfaction with mental health services is increasingly used as an outcome dimension and an indicator of service quality.

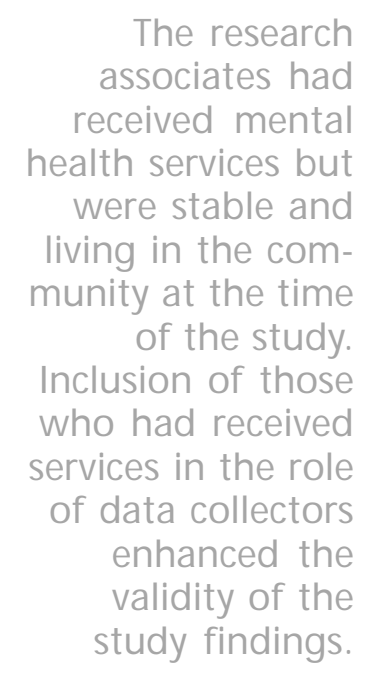

The research associates had received mental alth services but were stable and iving in the community at the time of the study. of those services in the role data collectors enhanced the study findings. 
Data analysis

U nivariate analysis was used to calculate means and standard deviations for continuous variables, and frequency distributions for catego rical variables.

\section{Despite the}

\section{current emphasis}

on patient-driven care in the United States, the

findings

suggested that

respondents did

not have input

into their

treatment

planning. In

addition, lack of

education about

medications and

treatment side-

effects are cause

for concern when

coupled with the

lack of

involvement of

family and friends

in the process of

treatment

planning.

\section{This study was funded by the United States Department of Health and Human Services,}

Substance Abuse and Mental Health Service

Administration, Center for Mental Health Services (Grant \# 4HRI SM 52058-02-4), Washington, DC, and the Kentucky

Department for

Mental Health and

Mental

Retardation

Services, Frankfort, Kentucky, USA.

\section{Results}

All the satisfaction scales demonstrated relatively high mean values when averaged across respondents. For example, the Q MHC item, which measured respondents' perceptions of the overall quality of their care, had an average score of 7.7 out of a possible score of 10 . For items on the KY-CSI and MHSIP, 70\% or more respondents indicated that they were satisfied with the services they had received during their hospital stay. The areas of greatest satisfaction were the time available to be with other patients $(94 \%)$ and the cleanliness of the facilities (92\%). In addition, staff-client relationships were a major source of satisfaction among respondents. Staff were reported to be available to speak with patients by $83 \%$ of respondents and $85 \%$ said they were comfortable talking to staff about their problems. Most respondents $(86 \%)$ reported that staff provided them with the information they needed to 'take charge' of their illnesses, and $84 \%$ said they received the services that they needed to get better. The majority of respondents $(87 \%)$ indicated that they understood what was expected of them during treatment, and that they perceived that staff had confidence in their ability to grow, change and recover $(86 \%)$ ). Furthermore, $84 \%$ of respondents reported that they felt better about themselves as a result of treatment.

D espite the overall high ratings of satisfaction, up to $20 \%$ of respondents reported some dissatisfaction with the services they received. For example, on the KY-CSI, $16.3 \%$ indicated that they would not return to the facility if they needed services in the future. Similarly, on the MH SIP, $19.6 \%$ of respondents indicated that if they had other options, they would not choose this health plan in the future. $0 \mathrm{n}$ the $\mathrm{KY}-\mathrm{CSI}, 13.8 \%$ of respondents reported that they did not receive medication education.

Treatment planning issues were a source of dissatisfaction for some respondents. N early $14 \%$ reported that staff did not ask them what they thought would help them get better, and $16.8 \%$ indicated that they did not feel free to complain. In addition, $16.8 \%$ of respondents indicated that their family and friends were not included in treatment planning. The physical environment was also a source of dissatisfaction; for example, $11.8 \%$ of respondents perceived a lack of privacy in the in-patient setting. Respondents also reported dissatisfaction with access to care; for example, $17.3 \%$ indicated that the location of services was inconvenient.

\section{The need for patient-driven care}

In general, the degree of satisfaction with mental health services appeared to be quite high. The findings suggested there had been a therapeutic, trusting and reciprocal relationship between staff and respondents. In addition, respondents reported high levels of satisfaction with staff-client relationships, staff availability and their ability to talk to staff members about their problems. Given the high degree of satisfaction with staff-client relationships and delivery of needed services, it is not surprising to find that the majority of respondents felt better about themselves as a result of treatment.

However, some items related to service dissatisfaction are noteworthy. Despite the current emphasis on patient-driven care in the United States, the findings suggested that respondents did not have input into their treatment planning. In addition, lack of education about medications and treatment side-effects are cause for concern when coupled with the lack of involvement of family and friends in the process of treatment planning (H oward et al, 2003). When patients and their carers do not understand treatment, or do not know about the sideeffects of medication, recovery is compromised and the consumer is at risk of relapse and readmission to hospital. Finally, the findings about areas of dissatisfaction raise questions about a continued emphasis on the provider's, rather than the client's, perspectives in the process of making decisions about the client's plan of care.

\section{References}

Attkinson, C. C. \& Greenfield, T. K. (1996) The Client Satisfaction Q uestionnaire (CSQ-8) and the Service Satisfaction Scale (SSS-30). In Outcomes Assessment in Clinical Practice (eds L. I. Sederer \& B. Dickey), pp. 120-128. Baltimore, MD: Williams and Wilkins.

Buckley, S. M. (1993) Moving MHSIP Towards a Person-Centered Paradigm. A concept paper submitted to CMHS and the MHSIP ad hoc advisory group. Available at www.mhsip.org/ documents/moving.htm. Last accessed 27 April 2004.

Campbell, J. (1998) Consumerism, outcomes, and satisfaction: a review of the literature. In Mental Health United States, 1998 (eds R. W. Manderscheid \& M. J. Henderson), pp. 11-28. Center for Mental Health Services, DHHS Publication N o. (SMA) 99-3285. Washington, DC: US Government Printing 0 ffice.

Center for Mental Health Services (1996) The MHSIP Consumer-Oriented Mental Health Report Card: Final Report of the Mental Health Statistics Improvement Program (MHSIP) Task Force on a Consumer-Oriented Mental Health Report Card. Rockville, MD: US Department of Health and Human Services, Public Health Service, Substance Abuse and Mental $\mathrm{H}$ ealth Services Administration.

Chisholm, M., Howard, P. B., Boyd, M. A., et al (1997) Q uality indicators for primary mental health within managed care: a public health focus. Archives of Psychiatric Nursing, 11, 167-181.

Ganju, V. (1999) The MHSIP Consumer Survey: H istory, Development, Revisions, Applications, Commonly-Asked Questions. Available at www.mhsip.org/documents/ MHSIPC onsumerSurvey.pdf. Last accessed 27 April 2004.

Howard, P. B. \& El-Mallakh, P. (2001) Training consumers to collect data in mental health service system evaluation research. Journal of Psychosocial Nursing and Mental Health Services, 39, 30-39.

Howard, P. B., Clark, J. J., Rayens, M. K., et al (2001) Consumer satisfaction with services in a regional psychiatric hospital: a collaborative research project in Kentucky. Archives of Psychiatric Nursing, 15, 10-23.

Howard, P. B., El-Mallakh, P., Rayens, M. K., et al (2003) Consumer perspectives on quality of inpatient mental health services. Archives of Psychiatric Nursing, 17, 205-217.

Teague, G. B., Ganju, V., Hornik, L. A., et al (1997) The MHSIP Mental Health Report Card: a consumer approach to monitoring the quality of mental heath plans. Evaluation Review, 21, 330-341. 